\title{
What are the temperatures of T Tauri stars?
}

\section{Constraints from coeval formation of young eclipsing binaries ${ }^{\star}$}

\author{
M. Ammler ${ }^{1}$, V. Joergens ${ }^{2}$, and R. Neuhäuser ${ }^{1}$ \\ 1 AIU Jena, Schillergäßchen 2-3, 07745 Jena, Germany \\ e-mail: ammler@astro.uni-jena.de \\ 2 Sterrewacht Leiden/ Leiden Observatory, Niels Bohrweg 2, 2333 CA Leiden, The Netherlands
}

Received 5 January 2005 / Accepted 24 June 2005

\begin{abstract}
We show how the assumption of coeval formation can be used to constrain the effective temperatures of the components of young eclipsing double-lined spectroscopic binaries. Our method extends the approach of White et al. (1999) to a two-step analysis. The first step compares evolutionary models to the observed masses and radii and selects those models that predict ages that are consistent with coeval formation. The second step then uses these models to constrain the effective temperatures. We applied the method to literature values of the stellar parameters of the eclipsing binaries RX J0529.4+0041 A and V1174 Ori and confirm that V1174 Ori A has dwarf-like temperatures at an age of 9 Myrs, while we cannot draw any conclusions for RX J0529.4+0041 A and V1174 Ori B. Considering these binaries, we find that none of the evolutionary models gives coeval solutions simultaneously in mass, radius and effective temperature.
\end{abstract}

Key words. stars: fundamental parameters - stars: atmospheres - Hertzsprung-Russell (HR) and C-M diagrams stars: pre-main sequence - stars: late-type

\section{Introduction}

Effective temperatures can be determined directly by measuring a star's angular diameter and its emerging total flux. While this works well for giant stars and very nearby main-sequence stars, the resolution of new interferometers now approaches also the red dwarf regime (Ségransan et al. 2003). Furthermore, effective temperatures can be derived with semi-direct methods, e.g. the infrared flux method which relies partly on stellar atmosphere modelling (see Blackwell et al. 1991). Fuhrmann (2004) determined precise effective temperatures of several hundred nearby stars by modelling the Balmer line wings. The application of these methods to T Tauri stars (TTSs) is difficult. The main problem is the relatively large distance to nearby star forming regions requiring very high angular resolution to measure diameters directly. Furthermore, in the case of classical TTSs, measurements are difficult because of the UV and IR emission from the accretion disk. Moreover, activity of TTSs results in strong spectral features which hamper the spectroscopic methods. Therefore, effective temperatures of TTSs usually have to be estimated by means of temperature calibrations.

In a few cases, the light curves of pre-main sequence (PMS) eclipsing double-lined spectroscopic binaries (SB2s) allow the

^ Appendices A and B are only available in electronic form at http://www. edpsciences.org direct determination of the ratio of the components' effective temperatures. At first there is no information on the individual temperatures of the components which have to be constrained by external information. The type of the external information generally depends on the binary studied and the available observational data. One common approach is the derivation of the primary temperature from its spectral type or colour index. As an example, Covino et al. (2004) estimated the effective temperature of RX J0529.4+0041 Aa from its spectral type by means of calibrations. The assumption of temperature scales for dwarf stars is supported by the surface gravities of the components (Covino 2005). Furthermore, they created a synthetic composite spectrum of RX J0529.4+0041 A which is based on the derived stellar parameters and the models of Hauschildt et al. (1999a,b). This spectrum was found to be consistent with the combined UBVRIJHK fluxes.

The derivation of the effective temperature from colour index or spectral type depends on the luminosity class, which is a priori unknown for PMS stars. Although the surface gravity can be precisely determined for PMS eclipsing SB2s, it remains unclear whether main-sequence or post main-sequence temperature scales are applicable to PMS stars. Therefore, we suggest a further constraint to be applied to PMS stars which follows White et al. (1999), and is based on the assumption of coevality and the use of evolutionary models. 
Their approach is the only method which determines effective temperatures of PMS stars independently of any assumptions on the luminosity class. They used the assumption of coeval components of the quadruple GG Tau to constrain their temperatures and find intermediate temperatures between giants and dwarfs. The method was applied by Luhman (1999) and Luhman et al. (2003) to create a temperature scale for intermediate luminosity classes at spectral types $\mathrm{M}$ for use with the evolutionary models of Baraffe et al. (1998) and Chabrier et al. (2000).

We extend the approach of White et al. (1999) in Sect. 2 to PMS eclipsing SB2s and apply it to RX J0529.4+00.41 A and V1174 Ori in Sect. 3.

\section{A new method - extending the approach of White et al. (1999)}

White et al. (1999) obtained the temperatures of the GG Tau components by comparing their luminosities to evolutionary models in the HR diagram. The assumption of coevality requires the components to be located on the same isochrones and therefore constrains their effective temperatures (see Sect. A.9.3 for further details). Although the temperatures are allowed to vary within a relatively large range between dwarf and giant values, coeval solutions are only found for the Baraffe et al. (1998) (BCAH98) isochrones. This coevality test is not independent of the derivation of the temperatures of the components. An independent test of the models (e.g. with individual masses and radii) cannot be done with GG Tau. In contrast to GG Tau, individual masses and radii of the components of eclipsing SB2s are well determined. Before constraining the effective temperatures, we can therefore first rule out all evolutionary models that do not give coeval solutions for the measured masses and radii. We retain only the models with coeval solutions supporting the accuracy of the resulting effective temperatures.

In our method we first compare the fundamental masses and radii of the binary components to the predictions of evolutionary models by D'Antona \& Mazzitelli (1994), D'Antona \& Mazzitelli (1997), BCAH98, Palla \& Stahler (1999) (PS99), Siess et al. (2000) and Yi et al. (2003) (see Table 1). Then, we keep only the models which give coeval solutions for masses and radii. As in White et al. (1999), the adopted temperatures may vary within a large range spanned by the various dwarf and giant scales as presented below. We obtain temperatures from each of these scales for the primaries and calculate the secondary temperatures from the temperature ratio given by the light curve analyses of Covino et al. (2004) and Stassun et al. (2004). Finally, we compare these temperatures to the selected evolutionary models in the $T_{\text {eff }}-R$ diagram to find consistent temperatures.

Compared to White et al. (1999), we apply a larger number of temperature scales. We compiled 14 effective temperature scales for several luminosity classes, focusing on spectral types G0-M 9. We consider these scales to be representative of the large number of temperature scales in the literature, and we believe that they reflect the range of physically possible temperatures at a certain spectral type.
Table 1. Overview of the used evolutionary models from D'Antona \& Mazzitelli (1994) (DM94), D'Antona \& Mazzitelli (1997) (DM97), BCAH98, PS99, Siess et al. (2000) (SDF00) and Yi et al. (2003) (YKD03) with the choices of free parameters. The table is restricted to those parameters which are necessary to distinguish between the models.

\begin{tabular}{l|llll}
\hline \hline model & metallicity & $Y$ & $X_{D} / 10^{-5}$ & Convection \\
\hline DM94 & & & & MLT \\
& & & & CM \\
\hline DM97 & $Z=0.01$ & 0.26 & $2 \times 10^{-5}$ & \\
& $Z=0.01$ & 0.26 & $4 \times 10^{-5}$ & \\
& $Z=0.01$ & 0.28 & $1 \times 10^{-5}$ & \\
& $Z=0.01$ & 0.28 & $2 \times 10^{-5}$ & \\
& $Z=0.01$ & 0.28 & $4 \times 10^{-5}$ & \\
\hline BCAH98 & {$[M / H]=0.0$} & 0.275 & & MLT $(\alpha=1.0)$ \\
& {$[M / H]=0.0$} & 0.275 & MLT $(\alpha=1.5)$ \\
& {$[M / H]=0.0$} & 0.282 & MLT $(\alpha=1.9)$ \\
& {$[M / H]=-0.5$} & 0.250 & MLT $(\alpha=1.0)$ \\
\hline PS99 & & 0.28 & MLT $(\alpha=1.5)$ \\
\hline SDF00 & $Z=0.01$ & 0.256 & & \\
& $Z=0.02$ & 0.277 & & +overshooting \\
& $Z=0.02$ & 0.277 & & \\
& $Z=0.03$ & 0.297 & & \\
& $Z=0.04$ & 0.318 & & \\
\hline YKD03 & $Z=0.01$ & & & \\
& $Z=0.023$ & & & \\
& $Z=0.03$ & & & \\
\hline
\end{tabular}

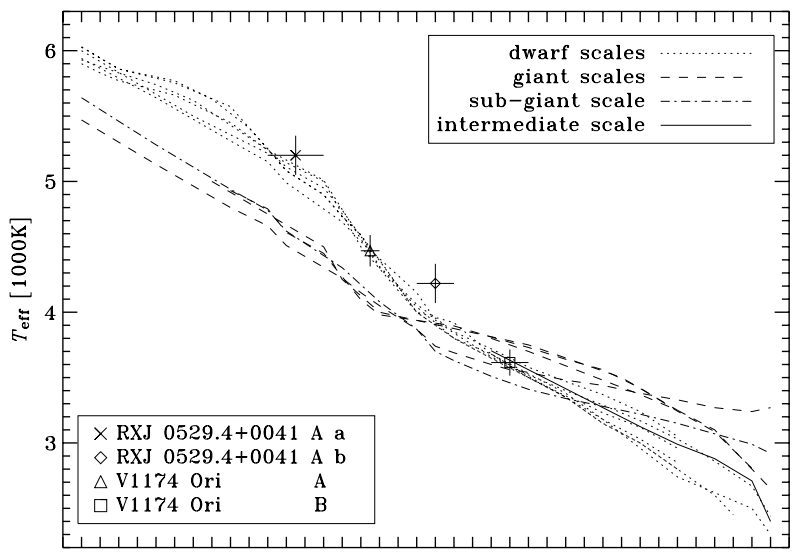

G0 G2 G4 G6 G8 K0K1.5K3 K5 K7 M0 M1 M2 M3 M4 M5 M6 M7 M8

Fig. 1. Overall view of the adopted temperature scales in the spectral type vs. effective temperature diagram. Additionally, the measured spectral types and effective temperatures of the eclipsing binaries are shown (from Table 3). The error bar of V1174 Ori B is only a very rough estimate.

Figure 1 displays an overview of these scales in a spectral type vs. effective temperature diagram. References and general information are listed in Table 2. Temperature values at each spectral type are tabulated for spectral types G0-M9 in Appendix B (Table B.1 for dwarf scales; Table B.2 for other scales). Furthermore, we compiled or derived intrinsic error estimates for the temperature scales (Table 2 and Appendix A). Figure 1 shows that the temperature scales for stars of spectral 
Table 2. Adopted temperature scales with validity range in MK spectral type, luminosity class, method of construction, intrinsic errors and validity range of the intrinsic errors. We distinguish between calibrations which are based directly on effective temperature measurements and those only derived from calibrations of other authors. We refer to the former as primary and to the latter as derived scales. Scales based on older calibrations but that account for new measurements are indicated by "improved". The origin of the intrinsic errors is explained in Appendix A.

\begin{tabular}{|c|c|c|c|c|c|}
\hline reference & sp. type & lum. class & construction & intrinsic errors & validity range of int. errors \\
\hline \multirow[t]{3}{*}{ Bessell $(1979, \text { Table } 2)^{1}$} & B7-M 6 & dwarfs & primary & $220 \mathrm{~K}^{2}$ & earlier G2 \\
\hline & & & & $\Delta \log T_{\mathrm{eff}}=0.035^{2}$ & later than $\mathrm{G} 2$ \\
\hline & & & & & and earlier than $\mathrm{K} 7$ \\
\hline Bessell $(1991 \text {, Table } 2)^{1}$ & K 7-M 7.5 & dwarfs & primary & $290 \mathrm{~K}^{2}$ & whole range \\
\hline Bessell (1979, Table 3) & G7-M 6 & giants & primary & $220 \mathrm{~K}^{2}$ & whole range \\
\hline Cohen \& Kuhi (1979, Table 7) & O9.5-M 6 & dwarfs & derived & $300 \mathrm{~K}^{2}$ & whole range \\
\hline de Jager \& Nieuwenhuijzen (1987, Table 5) & O3-M 9 & dwarfs & primary & $\Delta \log T_{\text {eff }}=0.021^{3}$ & whole range \\
\hline de Jager \& Nieuwenhuijzen (1987, Table 5) & O3-M 9 & subgiants & primary & $\Delta \log T_{\text {eff }}=0.021^{3}$ & whole range \\
\hline de Jager \& Nieuwenhuijzen (1987, Table 5) & O3-M 9 & giants & primary & $\Delta \log T_{\text {eff }}=0.021^{3}$ & whole range \\
\hline \multirow[t]{2}{*}{ Hartigan et al. (1994, Table 4) } & F0-M 6 & dwarfs & derived & $\Delta \log T_{\text {eff }}=0.015^{2}$ & earlier than $\mathrm{K} 7$ \\
\hline & & & & $290 \mathrm{~K}^{2}$ & later than $\mathrm{K} 7$ \\
\hline Kenyon \& Hartmann (1995, Table 5) & B0-M 6 & dwarfs & improved & $\Delta \log T_{\mathrm{eff}}=0.015^{2}$ & whole range \\
\hline \multirow[t]{4}{*}{ Perrin et al. (1998, Table 5) } & G8-M 8 & giants & primary & $220 \mathrm{~K}^{2}$ & earlier than M6 \\
\hline & & & & $79 \mathrm{~K}^{3}$ & M 6 \\
\hline & & & & $94 \mathrm{~K}^{3}$ & M7 \\
\hline & & & & $42 \mathrm{~K}^{3}$ & M 8 \\
\hline \multirow[t]{4}{*}{ Luhman (1999, Table 2) } & M 1-M9 & giants & derived & $270 \mathrm{~K}^{2}$ & earlier than $\mathrm{M} 7$ \\
\hline & & & & $100 \mathrm{~K}^{2}$ & M7 \\
\hline & & & & $50 \mathrm{~K}^{2}$ & M 8 \\
\hline & & & & $150 \mathrm{~K}^{2}$ & M9 \\
\hline Luhman et al. (2003, Fig. 8) & M 1-M 9 & dwarfs & improved & $80 \mathrm{~K}^{2}$ & whole range \\
\hline \multirow[t]{2}{*}{ Luhman et al. (2003, Table 8) } & M 1-M9 & intermediate & improved & $80 \mathrm{~K}^{2}$ & earlier than M 6.5 \\
\hline & & & & $100 \mathrm{~K}^{2}$ & later than M 6.5 \\
\hline Tokunaga (2000, Table 7.6) & O9-M 6 & dwarfs & derived & $100 \mathrm{~K}^{3}$ & later spectral types \\
\hline
\end{tabular}

${ }^{1}$ We only used a combination, i.e. Bessell (1979) for spectral types earlier than K7 and Bessell (1991) for spectral types later than K7.

${ }^{2}$ Intrinsic errors were derived by us or taken from another work.

${ }^{3}$ Intrinsic errors were adopted from the original work.

types earlier than $\mathrm{K} 7$ are divided into two distinct groups, one for the giants and one for the dwarfs, with giant temperatures being up to $\sim 500 \mathrm{~K}$ cooler than the dwarf temperatures. The spread in dwarf temperatures is up to $300 \mathrm{~K}$ for the same spectral type, and for giant temperatures it is $\sim 200 \mathrm{~K}$, giving a total span of physically possible effective temperatures at the same spectral types of up to $800 \mathrm{~K}$. At the spectral type $\sim \mathrm{K} 7$, we find a crossing point where all scales provide similar temperatures. At later spectral types the giant temperatures are generally hotter than the dwarf temperatures. The overall temperature spread increases again and peaks at $1000 \mathrm{~K}$ for the very late spectral types.

\section{Application to known eclipsing binaries}

\subsection{RXJ0529.4+0041 A and V1174 Ori}

In the past years, several eclipsing SB2s with low-mass PMS components have been found: RX J0529.4+0041 A (Covino et al. 2004), V1174 Ori (Stassun et al. 2004), TY CrA (Casey et al. 1998) and EK Cep (Hill \& Ebbighausen 1984;
Popper 1987). A further system with known masses, the astrometric binary NTT $045251+3016$, was found by Steffen et al. (2001). We use RX J0529.4+0041 A and V1174 Ori (see Table 3 for the adopted physical parameters) for further analysis. We do not consider TY CrA and EK Cep because their higher-mass primaries are already on the main sequence and therefore, coevality cannot be tested with the PMS evolutionary models ${ }^{1}$. Furthermore, we did not take into account NTT $045251+3016$ because the radii cannot be measured directly.

Similar to Covino et al. (2004), Stassun et al. (2004) determined the effective temperature of the primary on the grounds of its spectral type and the scale of Schmidt-Kaler (1982) while the temperature of the secondary was constrained by the temperature ratio from the light curve analysis.

While Fig. 1 shows that indeed the temperatures are consistent with the dwarf relations, it is puzzling that

\footnotetext{
${ }^{1}$ However, such high-mass main-sequence primaries enable the direct use of main-sequence temperature scales to determine the temperature of the lower-mass PMS secondary.
} 
Table 3. The physical parameters of the two young eclipsing binaries RX J0529.4+0041 A and V1174 Ori as adopted from Covino et al. (2004) and Stassun et al. (2004), respectively. We use their temperature solutions but also determine a set of additional effective temperatures using other temperature scales (see Sect. 3.3).

\begin{tabular}{l|ll}
\hline \hline & RX J0529.4+0041 Aa & RX J0529.4+0041 Ab \\
\hline$M\left[M_{\odot}\right]$ & $1.27 \pm 0.01$ & $0.93 \pm 0.01$ \\
$R\left[R_{\odot}\right]$ & $1.44 \pm 0.05$ & $1.35 \pm 0.05$ \\
$\log g[\mathrm{cgs}]$ & $4.22 \pm 0.02$ & $4.14 \pm 0.02$ \\
$T_{\text {eff }}[\mathrm{K}]$ & $5200 \pm 150$ & $4220 \pm 150$ \\
spectral type & $\mathrm{K} 1 \pm 1$ & $\mathrm{~K} 7-\mathrm{M} 0$ \\
\hline & $\mathrm{V} 1174$ Ori A & $\mathrm{V} 1174$ Ori B \\
\hline$M\left[M_{\odot}\right]$ & $1.009 \pm 0.015$ & $0.731 \pm 0.008$ \\
$R\left[R_{\odot}\right]$ & $1.339 \pm 0.015$ & $1.065 \pm 0.011$ \\
$\log g[\mathrm{cgs}]$ & $4.19 \pm 0.01$ & $4.25 \pm 0.01$ \\
$T_{\text {eff }}$ & $4470 \pm 120$ & $3615 \pm 100$ \\
spectral type & $\mathrm{K} 4.5 \pm 0.5$ & $\mathrm{M} 1.5$ \\
\hline
\end{tabular}

RX J0529.4+0041 Ab is somewhat hotter than both dwarf and giant temperatures. It is important to keep in mind here that these individual temperatures rely on much weaker constraints than the temperature ratio which is well known for both binaries from the eclipse light curves.

\subsection{First step - selecting appropriate evolutionary models}

For the case of V1174 Ori, Fig. 2 illustrates how we found evolutionary models giving coeval solutions for the radii. If a coeval solution can be found, we conclude that the specific set of models can be used to constrain the effective temperatures.

No single set of evolutionary models provides a coeval solution for the masses and radii of RX J0529.4+0041 Aa \& $\mathrm{Ab}$, so we do not consider this system any further. In the case of V1174 Ori, we find that only the metal-poor models of BCAH98 and the models of PS99 are consistent with coeval formation (Fig. 2). The corresponding age is $\sim 9$ Myrs. In contradiction to Stassun et al. (2004, Fig. 19), we do not find coeval solutions when using the models of Siess et al. (2000) ${ }^{2}$. Concerning RX J0529.4+0041 Ab, we point out that Covino et al. (2004) found inconsistencies between its rotational velocity and its radius. The synchronisation condition indicates that the measured radius is overestimated by about $20 \%$, possibly reconciling the age discrepancy with RX J0529.4+0041 Aa. Nevertheless, we give higher weight to the results of the light curve analysis at hand.

\subsection{Second step - constraining effective temperatures}

We now reconsider the effective temperatures of V1174 Ori A $\&$ B, the only system where evolutionary models provide coeval solutions in the previous section. We interpolate the primary's spectral type in each conversion table (Tables B.1

\footnotetext{
2 Their Fig. 19 suggests coevality if inspected by eye. However, using a more quantitative analysis by interpolating isochrones with small time steps, we find that coevality no longer holds.
}

and B.2) taking into account intrinsic errors of the particular scale (Table 2) and errors of the measured spectral types (Table 3). The spectral type of V1174 Ori A is earlier than M0 so that the scales of Luhman (1999) and Luhman et al. (2003) do not apply. The secondary's temperatures are calculated from the primary's temperatures and the measured temperature ratio. In order to compare with the predictions of the evolutionary models, we use the $T_{\text {eff }}-R$ diagram instead of the HR diagram because the radii of V1174 Ori A \& B are much better constrained than the luminosities, which are subject to systematic uncertainties such as extinction and distance. Figure 3 illustrates how we found temperatures consistent with the appropriate evolutionary tracks of BCAH98 and PS99.

These models suggest temperatures of $\sim 4500 \mathrm{~K}$ (BCAH98) and $4400 \mathrm{~K}$ (PS99), respectively, for V1174 Ori A. That means they rule out giant-like temperatures (cf. Fig. 3b) but do not distinguish between almost all the other scales. The subgiant scale of de Jager \& Nieuwenhuijzen (1987) yields temperatures which are inconsistent with the underabundant models of BCAH98 but consistent with the models of PS99. Also the temperature of V1174 Ori A from Stassun et al. (2004) is consistent with both models.

In the case of V1174 Ori B however, the two models do not agree with any temperature which we derived, not even with the temperature from Table 3 . The predicted temperatures are significantly hotter (by a few hundred K) than any of the derived dwarf or giant temperatures. Furthermore, coevality is no longer fulfilled in the $T_{\text {eff }}-R$ diagram. A similar effect was found by Hillenbrand \& White (2004) who systematically compared evolutionary models to PMS and main-sequence stars with known masses but not yet including V1174 Ori. Our results are mostly in line with the findings of Stassun et al. (2004) who compared all PMS stars with observationally determined masses to evolutionary models in both the HR diagram and the more fundamental mass-radius diagram.

\subsection{Discussion}

Our test in the current set-up still suffers from some shortcomings. First of all, we have only used $1 \sigma$ errors. Furthermore, we required strict coevality although the evolutionary models describe single stars and not close binaries. We do not know to what degree coevality is realised in real binaries. The formation of both components might have set in at slightly different times. Furthermore, magnetic fields and rotation are known to slow down star formation but are not taken into account in classical hydrostatic models, possibly causing some additional real or apparent relative age difference. Moreover, the hydrostatic models do not take into account the dynamical phases before the quasi-hydrostatic PMS contraction. Dynamical calculations by Wuchterl \& Tscharnuter (2003) predict differences in the contraction behaviour on the PMS at different stellar masses. In addition, theoretical luminosities and temperatures are based on stellar atmosphere models which still have to cope with several problems especially for young stars. Following Wuchterl (2001), Baraffe et al. (2002) and Wuchterl \& Tscharnuter (2003), one should not rely on classical hydrostatic models for ages of a few Myrs. 

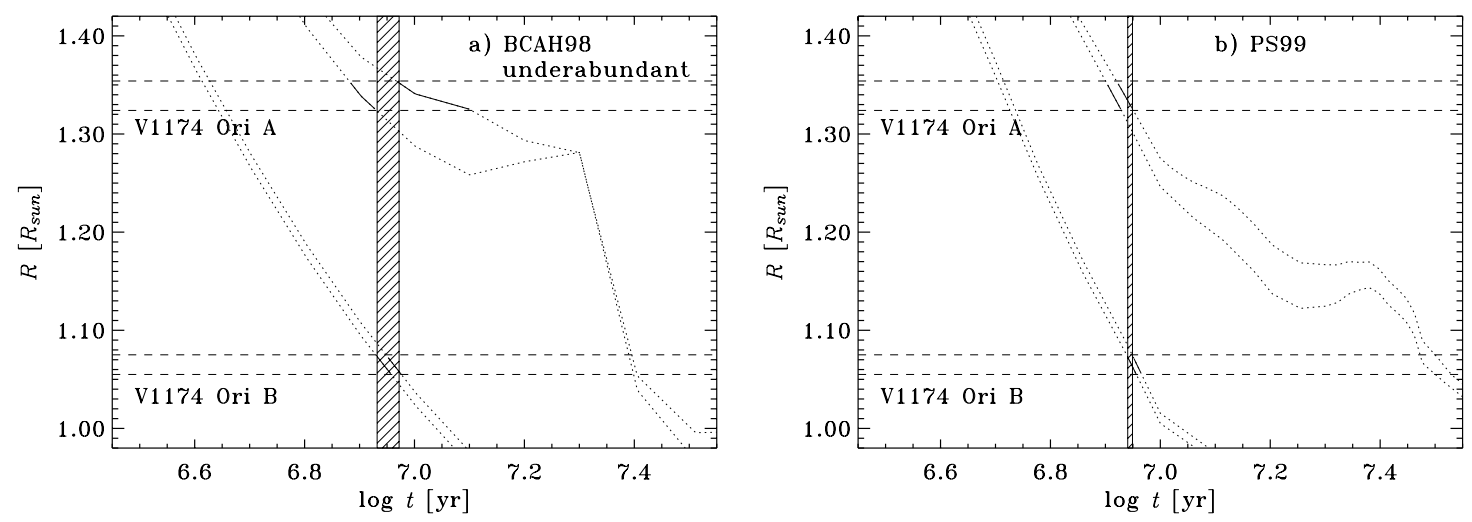

Fig. 2. The theoretical evolution of the radii of V1174 Ori A \& B is compared to the observed values. We only show the cases with coeval solutions: a) BCAH98, underabundant and b) PS99. The dashed horizontal lines indicate the upper and lower limits of the radius measurements. Evolutionary tracks for the upper and lower limits of the dynamical mass measurements were interpolated in the indicated models (dots). Solid lines represent the parts of the interpolated tracks which are consistent with the observed radii. These span the possible age of the individual binary components. The hatched region represents the coeval solution of $\sim 9$ Myrs.
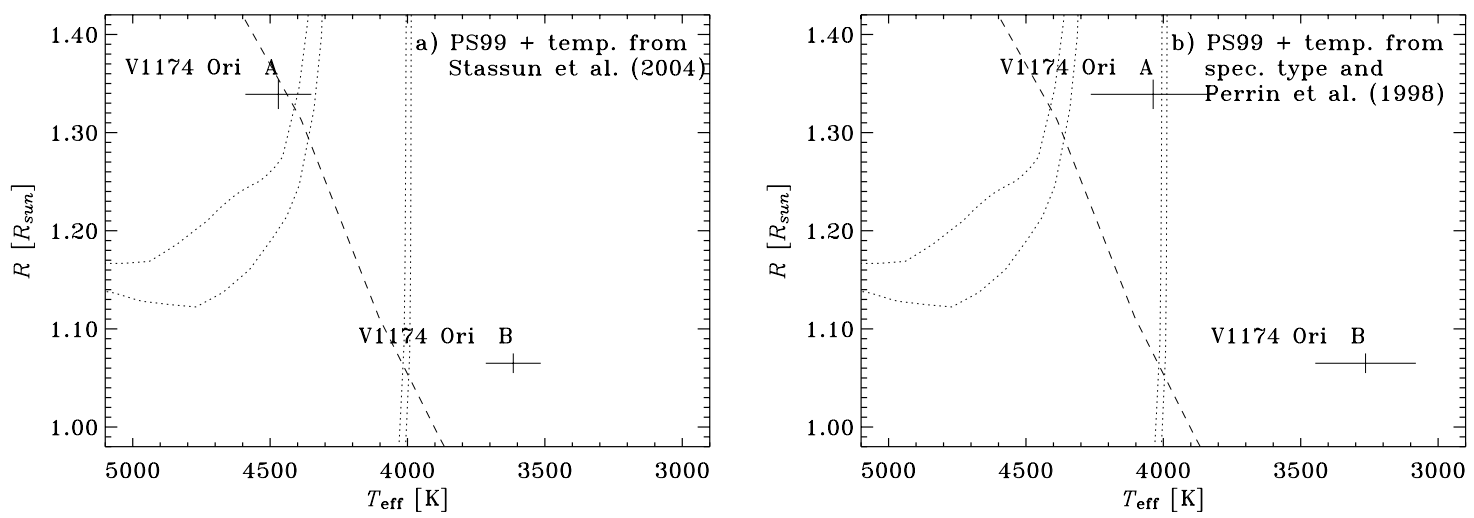

Fig. 3. $T_{\text {eff }}-R$ diagrams with the error bars of V1174 Ori A \& B. Evolutionary tracks for the upper and lower limits of the dynamical mass measurements (Table 3) (dots) were interpolated in the indicated tracks. The dashed line shows the theoretical isochrone at the common age of 9 Myrs found in Sect. 3.2. a) In this case, the temperatures were adopted from Table 3. The effective temperature of V1174 Ori A is consistent with the corresponding theoretical tracks whereas the temperature of V1174 Ori B is not. b) Temperatures were derived with the giant scale of Perrin et al. (1998) and are inconsistent with the models.

Moreover, a limited number of stellar parameters have been determined by observations for the systems considered here. Further observational constraints, e.g. on metallicities, should be included in order to find matching models. For example, it seems rather doubtful that of all the BCAH98 models, only the metal-poor model gives coeval radius solutions for V1174 Ori. Of course, these shortcomings also apply to all such comparisons which have been performed by other authors.

\section{Summary and conclusions}

Up to now, precise temperature constraints for PMS stars are only available from PMS eclipsing SB2s. While the effective temperature ratio can be precisely determined from the light curves, individual temperatures of each component are based on more uncertain external constraints. Therefore, we propose in this paper an extension of the method of White et al. (1999) to constrain effective temperatures of the components of PMS eclipsing SB2s. The application of our method is at the moment restricted to the PMS eclipsing SB2s RX J0529.4+0041 A and V1174 Ori.
The method first compares the empirical masses and radii of the binary components to the predictions of evolutionary models and selects those models which give coeval solutions. Secondly, a set of temperatures is derived from the primary's spectral type using several temperature scales. Then, temperatures for the secondary are calculated from each primary temperature and the temperature ratio, which is known from the light curve analysis. Finally, these temperatures are compared to the predictions of the selected models in the $T_{\text {eff }}-R$ diagram.

For the second step of the procedure, we use our compilation of temperature scales for dwarfs, giants and intermediate luminosity classes from the literature. If not yet available, we derived intrinsic uncertainties of the scales. The scales are different due to the different derivation methods. Even scales for the same luminosity class differ by up to $300 \mathrm{~K}$ at spectral types $\mathrm{G}$ and up to $1000 \mathrm{~K}$ at late-M types. Intrinsic uncertainties in the individual temperature scales typically amount to a few hundred K.

Applying the first step of our analysis to the PMS binaries, we find that none of the models is consistent with coevality of 
RX J0529.4+0041 Aa \& Ab while coevality of V1174 Ori A \& B is only consistent with the Palla \& Stahler (1999) and the metal-poor Baraffe et al. (1998) models. However, it seems doubtful that V1174 Ori is that metal-poor.

In the second step, neither the dwarf nor the giant temperatures nor the temperatures estimated by Stassun et al. (2004) are consistent with the models in the case of V1174 Ori B. V1174 Ori A alone gives strong evidence for dwarf-like temperatures at spectral types mid-K and ages of $\geq 9$ Myrs corroborating the use of dwarf scales for RX J0529.4+0041 A by Covino et al. (2004) and for V1174 Ori by Stassun et al. (2004).

As in the studies by Luhman (1999) and Luhman et al. (2003), our method may provide a new intermediate temperature scale for young stars once enough accurate stellar parameters from PMS eclipsing binaries are available.

Acknowledgements. We are grateful for the useful suggestions by G. Wuchterl and want to thank K. Fuhrmann and T. Gehren for the interesting discussions on effective temperature determination. We thank E. Covino, Th. Schmidt-Kaler, and S. Kenyon for giving useful remarks. We further thank G. Torres, F. Comerón, G. Herbig, and A. Richichi for reading the paper and giving interesting suggestions and, in particular, G. Torres for significantly improving the English. We are further thankful for useful comments by H.-G. Ludwig. M.A. acknowledges financial support from the episcopal study foundation "Cusanuswerk". V.J. acknowledges support from the Deutsche Forschungsgemeinschaft (Schwerpunktprogramm "Physics of star formation") and from the European community by a Marie Curie Individual Fellowship. M.A., V.J. and R.N. did part of this work when they were together at MPE Garching in 2001/2002.

\section{References}

Allard, F., \& Hauschildt, P. 1995, ApJ, 445, 433

Baraffe, I., Chabrier, G., Allard, F., \& Hauschildt, P. 1998, A\&A, 337, 403, (BCAH98)

Baraffe, I., Chabrier, G., Allard, F., \& Hauschildt, P. H. 2002, A\&A, 382,563

Barnes, T., Evans, D., \& Moffett, T. 1978, MNRAS, 183, 285

Bell, R., \& Gustaffson, B. 1989, MNRAS, 236, 653

Bessell, M. 1979, PASP, 91, 598

Bessell, M. 1991, AJ, 101, 662

Bessell, M., \& Brett, J. 1988, PASP, 100, 1134

Blackwell, D., Lynas-Gray, A., \& Petford, A. 1991, A\&A, 245, 567

Böhm-Vitense, E. 1981, ARA\&A, 19, 295

Böhm-Vitense, E. 1982, ApJ, 255, 191

Burgasser, A. J., Kirkpatrick, J. D., Brown, M. E., et al. 2002, ApJ, 564,421

Casey, B. W., Mathieu, R. D., Vaz, L. P. R., Andersen, J., \& Suntzeff, N. B. 1998, AJ, 115, 1617

Chabrier, G., Baraffe, I., Allard, F., \& Hauschildt, P. 2000, ApJ, 542, 464

Code, A., Davis, J., Bless, R., \& Hanbury Brown, R. 1976, ApJ, 203, 417

Cohen, M., \& Kuhi, L. 1979, ApJS, 41, 743

Conti, P. 1973, ApJ, 179, 181

Covino, E. 2005, priv. comm.

Covino, E., Frasca, A., Alcalá, J. M., Paladino, R., \& Sterzik, M. F. 2004, A\&A, 427, 637

D’Antona, F., \& Mazzitelli, I. 1994, ApJS, 90, 467 (DM94)
D’Antona, F., \& Mazzitelli, I. 1997, in Mem. S. A. It., Vol. 68, Cool stars in Clusters and Associations, ed. T. Pallavicini, \& G. Micela, No. 4, (DM97)

de Jager, C. 1980, The Brightest Stars (Dordrecht: Reidel), 24, 78, 84, 113,293

de Jager, C., \& Nieuwenhuijzen, H. 1987, A\&A, 177, 217

Fuhrmann, K. 2004, AN, 325, 3

Guilloteau, S., Dutrey, A., \& Simon, M. 1999, A\&A, 348, 570

Hartigan, P., Strom, K., \& Strom, S. 1994, ApJ, 427, 961

Hauschildt, P. H., Allard, F., \& Baron, E. 1999a, ApJ, 512, 377

Hauschildt, P. H., Allard, F., Ferguson, J., Baron, E., \& Alexander, D. R. 1999b, ApJ, 525, 871

Hayes, D. 1978, in The HR Diagram, ed. A. Philip, \& D. Hayes, IAU Symp., 80, 65

Henry, T., Kirkpatrick, J., \& Simons, D. 1994, AJ, 108, 1437

Hill, G., \& Ebbighausen, E. G. 1984, AJ, 89, 1256

Hillenbrand, L. A., \& White, R. J. 2004, ApJ, 604, 741

Johnson, H. 1965, ApJ, 141, 170

Johnson, H. 1966, ARA\&A, 4, 193

Jones, H., Longmore, A., Allard, F., et al. 1995, MNRAS, 277, 767

Kenyon, S. 2001, priv. comm.

Kenyon, S., \& Hartmann, L. 1995, ApJS, 101, 117

Leggett, S. 1992, ApJS, 82, 351

Leggett, S., Allard, F., Berriman, G., Dahn, C., \& Hauschildt, P. 1996, ApJS, 104, 117

Leggett, S., Allard, F., \& Hauschildt, P. 1998, ApJ, 509, 836

Leggett, S. K., Allard, F., Dahn, C., et al. 2000, ApJ, 535, 965

Luhman, K. 1999, ApJ, 525, 466

Luhman, K., \& Rieke, G. 1998, AJ, 497, 354

Luhman, K., Stauffer, J., Muench, A., et al. 2003, ApJ, 593, 1093

Palla, F., \& Stahler, S. 1999, ApJ, 525, 772, (PS99)

Perrin, G., Coudé du Foresto, V., Ridgway, S., et al. 1998, A\&A, 331, 619

Popper, D. 1980, ARA\&A, 18, 115

Popper, D. M. 1987, ApJ, 313, L81

Richichi, A., Ragland, S., Stecklum, B., \& Leinert, C. 1998, A\&A, 338,527

Ridgway, S., Joyce, R., White, N., \& Wing, R. 1980, ApJ, 235, 126

Schmidt-Kaler, T. 1982, in Landolt-Börnstein, ed. K. Schaifers, \& H. Voigt, Vol. 2 (Berlin: Springer), 449

Schmidt-Kaler, T. 2001, priv. comm.

Ségransan, D., Kervella, P., Forveille, T., \& Queloz, D. 2003, A\&A, 397, L5

Siess, L., Dufour, E., \& Forestini, M. 2000, A\&A, 358, 593

Stassun, K., Mathieu, R., Vaz, L., Stoud, N., \& Vrba, F. 2004, ApJS, 151,357

Steffen, A. T., Mathieu, R. D., Lattanzi, M. G., et al. 2001, AJ, 122, 997

Straizy̌s 1992, Multicolour Stellar Photometry (Tucson: Pachart)

Tokunaga, A. 2000, in Allen's Astrophysical Quantities, 4th ed., ed. A. N. Cox (New York: Springer), 143

van Belle, G., Lane, B., Thompson, R., et al. 1999, ApJ, 117, 521

Vardya, M. 1970, ARA\&A, 8, 87

Veeder, G. 1974, AJ, 79, 1056

White, R., Ghez, A., Reid, I., \& Schulty, G. 1999, ApJ, 520, 811

Wing, R., \& Yorka, S. 1979, in Ric. Astron. Specola Vaticana, Spectral Classification of the Future, ed. M. McCarthy, A. Philip, \& G. Coyne, 9, 519

Wuchterl, G. 2001, in The Formation of Binary Stars, ed. H. Zinnecker, \& R. Mathieu, IAU Symp., 200, 492

Wuchterl, G., \& Tscharnuter, W. M. 2003, A\&A, 398, 1081

Yi, S. K., Kim, Y., \& Demarque, P. 2003, ApJS, 144, 259 
M. Ammler et al.: What are the effective temperatures of T Tauri stars?, Online Material p 1

\section{Online Material}




\section{Appendix A: Derivation of intrinsic scale errors}

\section{A.1. Bessell $(1979,1991)$}

Bessell (1979) created temperature scales for dwarfs and giants (their Tables 2 and 3, resp.). The scale for $\mathrm{M}$ dwarfs has been improved by Bessell (1991) so that we consider only the combined dwarf scale (see Table 2). For spectral types earlier than K7 the dwarf scale of Bessell (1979, Table 2) is applied and for later types the work of Bessell (1991).

\section{A.2. Bessell (1979)}

Bessell (1979) does not give any errors for the temperature scales. In order to get an estimation for these errors, we analyse the origin of the temperatures in more detail.

The dwarf temperature scale is based upon a fit-by-eye to the temperatures of Code et al. (1976) (cf. Bessell 1979, Table 1, Fig. 10) for early spectral types. The independent variable in the fit is not the spectral type but the Strömgren index $(b-y)$ which has to be converted to spectral type. The temperatures derived by Code et al. (1976) and the fit in Bessell (1979) allow us to calculate the standard deviation. This value of $\Delta \log T=0.035$ dex now represents an estimate for the uncertainties of the scale for early spectral types. We did not take into account any errors that might be introduced by the conversion from $(b-y)$ to spectral type.

The temperature scale for dwarfs for temperatures between $4000 \mathrm{~K}$ and the temperature of the sun is based on the relation between $(V-I)$ and the temperatures of giants.

The temperatures for cooler dwarfs are taken from the work of Veeder (1974) who fitted black body distributions to spectral energy distributions between $0.4 \mu \mathrm{m}$ and $3.5 \mu \mathrm{m}$. Those temperatures are not used in our work because they have been superseded by Bessell (1991).

The temperatures for giants cooler than the sun were adopted from the scale of Ridgway et al. (1980). In order to derive their temperature scale, Ridgway et al. (1980) only used direct effective temperature measurements with errors lower than $\pm 250 \mathrm{~K}$ (cf. their Table 3 ) and performed a fit-by-eye in the $\left(T_{\text {eff }}-T_{\mathrm{C}}\right)$ diagram. The colour temperatures $T_{\mathrm{C}}$ resulted from a fit of black body distributions to the continuum flux at $8500 \AA$ and $10500 \AA$. The colour temperatures have been converted into spectral types (Wing \& Yorka 1979) in order to obtain a relation between spectral type and effective temperature. The work of Ridgway et al. (1980) does not provide any uncertainties for the temperature scale. In order to get an idea of the uncertainty of the scale in Bessell (1979), we calculated the standard deviation of the fit-by-eye and obtained $220 \mathrm{~K}$. Approximating the fit-by-eye with a second degree polynomial yields a similar result, when excluding the problematic stars BS 5301, HD 75156 and HD 29051. We did not take into account any errors which might be introduced by the conversion from $T_{\mathrm{C}}$ to spectral type.

\section{A.3. Bessell (1991)}

Bessell (1991) combined in their Fig. 11 their own colour measurements and effective temperatures from the literature in the $(R-I)-T_{\text {eff }}$ diagram for early-type stars and in the $(I-K)-T_{\text {eff }}$ diagram for late-type stars. The fundamental temperatures of YY Gem and CM Dra were included. The data were approximated by third order polynomials. The fundamental temperatures obviously have significantly higher weight. The origin of the spectral types in their Table 2 was not clarified in Bessell (1991).

Bessell (1991) do not provide any errors for the temperature scale. In order to get an estimate for the uncertainties implied in the application of this scale, we calculated the standard deviation of the polynomial $(R-I)$ calibration for stars in their Fig. 11a. It amounts to $290 \mathrm{~K}$. We used only some 20 stars for which we could reproduce the required data following the information given in Bessell (1991). We adopt the calculated standard deviation also for the later spectral types as is justified by a short inspection by eye of their Fig. 11 b.

\section{A.3.1. Summary: the intrinsic errors}

The application of the giant scale implies an error of $220 \mathrm{~K}$ at all spectral types. The error for the combined dwarf temperature scale is approximately $\Delta \log T_{\text {eff }}=0.035 \mathrm{dex}$ for spectral types earlier than that of the sun, $\pm 220 \mathrm{~K}$ for later types earlier than K7 and $\pm 290 \mathrm{~K}$ for the latest types. We consider both scales to be primary scales because they were constructed directly with temperatures of individual stars.

\section{A.4. Cohen \& Kuhi (1979)}

Cohen \& Kuhi (1979) created a temperature scale (their Table 7) which was recommended by Schmidt-Kaler (1982) for the analysis of pre-main sequence stars. It is based on scales from the literature. Though Cohen \& Kuhi (1979) found that the luminosity class of $\mathrm{T}$ Tauri stars is between that of giants and dwarfs, they use a temperature scale which is based on dwarf scales because those were available more easily. Thus, to be consistent, we consider this temperature scale to be a dwarf scale. According to Cohen \& Kuhi (1979) the adoption of this scale for T Tauri stars implies an overestimation of the temperatures by $5 \%$. We find that the temperatures would be overestimated only for spectral types earlier than $\mathrm{M}$ if we would assign giant-like properties to T Tauri stars.

The temperature scale of Cohen \& Kuhi (1979) is based on the work of Vardya (1970) who applied temperatures of Johnson (1965) and Johnson (1966), inferred from observed apparent diameters and bolometric fluxes. The temperatures for the spectral types $\mathrm{K} 5$ and $\mathrm{K} 7$ are obviously adopted from Johnson (1966) and for M from Vardya (1970). Temperatures for fractional spectral types and for K7-M0 are apparently found by interpolation.

Cohen \& Kuhi (1979) do not provide estimates for the uncertainties of their temperature scale. Therefore we estimated the uncertainties by considering the work of Johnson (1965). The standard deviation of the fit in Johnson (1965, Fig. 4), 
approximately $300 \mathrm{~K}$, was found by eye. This is adopted as uncertainty for this work. We consider the scale of Cohen \& Kuhi (1979) to be a derived scale.

\section{A.5. de Jager \& Nieuwenhuijzen (1987)}

Using a large number of stars with known luminosities and effective temperatures, de Jager \& Nieuwenhuijzen (1987) determined the statistical dependencies of luminosity and effective temperature on spectral type and luminosity class, respectively. Spectral types and luminosity classes are represented by continuous variables. A large number of stellar temperatures was approximated by Chebychev polynomials in the continuous variables. The resulting temperatures are tabulated in their Table 5 .

For the application to the analysis of T Tauri stars, we extracted the approximated temperatures for dwarfs, subgiants and giants separately from their Table 5. The standard deviation of the fit in de Jager \& Nieuwenhuijzen (1987) is $\Delta \log T_{\text {eff }}=0.021$, as given by the authors. This value is used as the intrinsic error of the obtained dwarf, subgiant and giant scales.

\section{A.6. Hartigan et al. (1994)}

According to the authors, the scale in their Table 4 is based on the work of Bessell \& Brett (1988) and Schmidt-Kaler (1982). However, temperatures could not be found in the former. For K7 and $\mathrm{M}$ types they are identical to the temperatures in Bessell (1991, Table 2) (cf. Sect. A.3). For types earlier than K7, the dwarf scale of Schmidt-Kaler (1982) was adopted with the adopted value at $\mathrm{K} 5$ being higher by $50 \mathrm{~K}$.

Consequently, as Hartigan et al. (1994) do not provide intrinsic errors for their scale, we adopt the errors of the combined dwarf scale of Bessell (1979) and Bessell (1991) and the dwarf scale of Kenyon \& Hartmann (1995), the latter being based on Schmidt-Kaler (1982) (see Sect. A.7). The intrinsic error of the scale in Bessell (1991), $\pm 290 \mathrm{~K}$, is adopted for K7 and $\mathrm{M}$ spectral types and $\Delta \log T_{\mathrm{eff}}= \pm 0.015$ for earlier types, according to Sect. A.7. We consider the scale of Hartigan et al. (1994) to be a derivation of the scales of Schmidt-Kaler (1982) and Bessell (1991).

\section{A.7. Kenyon \& Hartmann (1995), Schmidt-Kaler (1982)}

The effective temperatures in Kenyon \& Hartmann (1995, Table 5) are based on the temperatures given in Schmidt-Kaler (1982, Table 3, p. 453). The latter was derived as described in the following (Schmidt-Kaler 2001): direct fundamental data were interferometric measurements of stellar diameters (Table 22, p. 30 in Schmidt-Kaler 1982; de Jager 1980, Table 10), the sun and de Jager (1980, Table 6). Interpolation in spectral type yielded a relation between spectral type and radii (Schmidt-Kaler 1982, Table 23, p. 31). Surface brightnesses were added and also interpolated in spectral type. This result was then compared and corrected with the literature. The smoothed result is shown in Schmidt-Kaler (1982, Table 3, p. 453). For accuracy reasons, $(U-B)_{0}$ instead of spectral type was used as parameter for very early-type stars, but then transformed to spectral types. Similarly $(R-I)_{0}$ was applied for late-M type stars. In the range B8-K3 V/III, the error is $\Delta \log T_{\text {eff }} \approx \pm 0.015$. For O3-O6 and M5-M 8 the errors are much larger.

Following Kenyon (2001), the effective temperature scale of Kenyon \& Hartmann (1995) is based on integrated spectral energy distributions which are generated by means of the colours in Table 5 of Kenyon \& Hartmann (1995). Ultraviolet magnitudes are included to obtain reliable spectral energy distributions. If the derived temperatures are close enough to those of Schmidt-Kaler (1982) and Straizy̌s (1992), an appropriate average applies. In case of discontinuities of the scales of Schmidt-Kaler (1982) and Straizy̌s (1992) and simultaneous discrepancy with the temperatures, that originate from the spectral energy distributions, the latter are favoured.

For all spectral types with temperatures given in SchmidtKaler (1982, Table 3, p. 453), we actually find the same temperatures in Kenyon \& Hartmann (1995) except for G8, with the temperature being lower by $50 \mathrm{~K}$ in Kenyon \& Hartmann (1995).

In their appendix B, Kenyon \& Hartmann (1995) provide a relative error of 5 to $10 \%$ for their entire Table 5 affecting colours, effective temperatures and bolometric corrections. However Kenyon (2001) recommends to obtain errors from Schmidt-Kaler (1982) and Straizy̌s (1992). The error $\Delta \log T_{\text {eff }} \pm 0.015$ (Schmidt-Kaler 2001) is applied in our work. We deem Kenyon \& Hartmann (1995, Table 5) to be an improvement of the work of Schmidt-Kaler (1982).

\section{A.8. Perrin et al. (1998)}

Perrin et al. (1998) extend the effective temperature scale for giants to spectral types later then M 6 using interferometrically determined radii of nine giant stars. The temperature scale is tabulated in their Table 5 .

The temperatures for spectral types earlier than M6 are based on the work of Ridgway et al. (1980) (cf. Sect. A.2). These are considered to be most consistent with previous effective temperature scales and the Perrin et al. (1998) data. Correspondingly, for the earlier spectral types we applied an error of $\pm 220 \mathrm{~K}$.

Perrin et al. (1998) derived temperatures for spectral types later than M 6. The temperature for M 6 is identical to the effective temperature of EU Del. For M 8, the average of the temperatures of SW Vir and RX Boo is adopted. The value for M7 is found by interpolation. The resulting errors are $\pm 79 \mathrm{~K}$ for $\mathrm{M} 6$, $\pm 94 \mathrm{~K}$ for $\mathrm{M} 7$ and $\pm 42 \mathrm{~K}$ for $\mathrm{M} 8$. Though those errors are only individual errors of single star and any possible statistical scatter is not accounted for, we adopt those errors for consistency reasons. The scale is based on temperatures of individual stars and can be regarded as a primary scale. 


\section{A.9. Luhman (1999); Luhman et al. (2003)}

Luhman (1999) provides in Table 2 effective temperature scales for dwarfs, giants and intermediate luminosity classes for spectral types later than M0. The dwarf and intermediate scales were updated by Luhman et al. (2003, Table 8). No errors are given for those temperature scales and were estimated by us as explained below.

\section{A.9.1. Dwarf scale}

The dwarf scale in Luhman (1999) is based on Luhman \& Rieke (1998) who linearly fitted data from Leggett et al. (1996). Moreover, the scale in Luhman (1999) is consistent with modelling results of Leggett et al. (1998). For the assessment of errors we considered the work of Leggett et al. (1996). They derived effective temperatures of low-mass stars by fitting synthetic spectra (Allard \& Hauschildt 1995) to observed low-resolution spectra. Spectral types come from Henry et al. (1994), Leggett (1992) and Boeshaar \& Liebert. The errors of the temperatures are $\pm 150 \mathrm{~K}$ and $\pm 250 \mathrm{~K}$, respectively (Leggett et al. 1996, Table 7).

Luhman et al. (2003) improved this temperature scale by adjusting the temperatures to be consistent with the latest temperature estimates for young disk dwarfs (Leggett et al. 2000; Burgasser et al. 2002): $-50 \mathrm{~K}$ at M 5 and $-100 \mathrm{~K}$ at M 6-M 9 (see their Fig. 8).

The Leggett et al. (1996) data allowed us to calculate the standard deviation of the fit in Luhman \& Rieke (1998). We consider this value of $\pm 80 \mathrm{~K}$ an overall estimate for the uncertainty of the dwarf scale of Luhman (1999) and Luhman et al. (2003).

\section{A.9.2. Giant scale}

The giant scale in Luhman (1999) is adopted from van Belle et al. (1999) for spectral types earlier than M 7, from Perrin et al. (1998) (see Sect. A.8) for spectral types M 7 and M 8, and from Richichi et al. (1998) for spectral type M9. Richichi et al. (1998) and van Belle et al. (1999) inferred effective temperatures directly by using apparent angular diameters that originate from lunar occultation measurements and interferometric measurements, respectively.

Van Belle et al. (1999) provide the standard deviation $270 \mathrm{~K}$ of a linear fit as an estimation of the uncertainty of their scale. The corresponding errors of the giant scale of Luhman (1999) are $270 \mathrm{~K}$ for spectral types earlier than M 7, $\pm 100 \mathrm{~K}$ for M 7 , $\pm 50 \mathrm{~K}$ for $\mathrm{M} 8$ and $\pm 150 \mathrm{~K}$ for $\mathrm{M} 9$. We consider this scale a derived scale.

\section{A.9.3. Intermediate scale}

Luhman (1999) created an intermediate temperature scale from the temperatures and spectral types of the GG Tau components. The temperatures were obtained by following the steps of White et al. (1999), comparing the luminosities of the components to evolutionary models in the HR diagram. The assumption of coevality requires the components to be located on the same isochrones. Therefore, their effective temperatures were allowed to vary in order to find a coeval solution (see Sect. 2 for a discussion). With correct temperatures all components should be placed on the same model isochrone. This isochrone is fixed by GG Tau Aa \& Ab with spectral types K7 and M0.5, respectively, as giant temperatures and dwarf temperatures are not very different in this range of spectral types. A further constraint is the total mass of GG Tau A which was determined by Guilloteau et al. (1999) from the Keplerian rotation of the circumbinary disk. An evolutionary model is then considered to be consistent with GG Tau if GG Tau A fulfils the mass constraint and if the fixed isochrone yields a solution for the temperatures of the low-mass components GG Tau Ba $\& \mathrm{Bb}$.

Luhman (1999) found coevality of all GG Tau components when using Baraffe et al. (1998) isochrones and effective temperatures of $3057 \mathrm{~K}$ and $2805 \mathrm{~K}$ for GG Tau Ba and Bb at spectral types M 5.5 and M 7.5, respectively. The temperature scale was extrapolated from M 5.5 to M0 $(3850 \mathrm{~K})$. Temperatures for M 8 and M 9 are chosen to be intermediate between giants and dwarfs and to provide continuity of the intermediate scale.

The intermediate scale of Luhman (1999) is only valid for M stars. For young stars with earlier spectral types, an intermediate scale is not deemed to be necessary because they evolve quickly towards the main sequence. Their luminosity class is only for a short time between that of giants and dwarfs, whereas M stars develop much slower and have an intermediate character for a correspondingly longer time span. Our analysis allows to check this assumption as we show in Sect. 3.

Using White et al. (1999), we assessed intrinsic errors for the intermediate scale of Luhman (1999). White et al. (1999) provide error bars for the effective temperatures of the GG Tau components. Those errors are attributed to uncertainties in the spectral types of the components: \pm 0.5 subclasses for $\mathrm{Ba}$ and $\mathrm{Bb}$. Direct assessment of the effective temperature error bars in White et al. (1999, Fig. 6) by eye yielded $\pm 80 \mathrm{~K}$ and $\pm 100 \mathrm{~K}$, resp. The uncertainties of the evolutionary models should also be considered since the intermediate temperature scale is created by means of evolutionary models. The comparison of the Baraffe et al. (1998) models for $l / H_{\mathrm{P}}=1.9$ and $l / H_{\mathrm{P}}=1.0$ should provide a simple upper limit for error estimates. Corresponding to White et al. (1999), coevality of the GG Tau components using the model with $l / H_{\mathrm{P}}=1.0$ yields effective temperatures of $3160 \mathrm{~K}$ and $2840 \mathrm{~K}$ for GG Tau Ba and $\mathrm{Bb}$, respectively. That differs by $110 \mathrm{~K}$ and $20 \mathrm{~K}$, resp. from the values derived with the model for $l / H_{\mathrm{P}}=1.9(3050 \mathrm{~K}$ and $2820 \mathrm{~K}$, respectively). This difference is of the same order as the uncertainties derived from the spectral types or even lower. Hence, the errors which originate from the uncertainties of the spectral types alone should provide a rough estimate for the intrinsic errors of the intermediate temperature scale of Luhman (1999). We adopt $\pm 80 \mathrm{~K}$ for spectral types M earlier than M 6.5 and $\pm 100 \mathrm{~K}$ later than $\mathrm{M} 6.5$.

Luhman et al. (2003) improved the intermediate temperatures of Luhman (1999) by adjustments at spectral types M7-9, so that the sequences of IC 348 and Taurus are coeval in the Hertzsprung-Russell diagram when comparing to the 
evolutionary models of Baraffe et al. (1998) and Chabrier et al. (2000). For this adjustment, the temperatures were reduced by $10 \mathrm{~K}$ at $\mathrm{M} 7$ and $\mathrm{M} 8$ and by $150 \mathrm{~K}$ at $\mathrm{M} 9$.

\section{A.10. Tokunaga (2000)}

The dwarf temperature scale of Tokunaga (2000) is an average of scales of other authors and therefore considered to be a derived scale within the frame of this work. For the relevant late spectral types the scale results from averaging the values of Popper (1980), Böhm-Vitense (1981), Böhm-Vitense (1982), Blackwell et al. (1991), Bell \& Gustaffson (1989), Bessell (1991), Jones et al. (1995) and Leggett et al. (1996). An intrinsic error of $\pm 100 \mathrm{~K}$ is provided for the later spectral types.

Popper (1980) uses temperatures of Hayes (1978) and fluxes of Barnes et al. (1978). The temperatures in Hayes (1978) are based on Code et al. (1976) (cf. Sect. A.2) and Conti (1973). Böhm-Vitense (1981) provides temperatures based on different publications. Blackwell et al. (1991) derive temperatures with the infrared flux method and improved $\mathrm{H}^{-}$-opacities. Bell \& Gustaffson (1989) use the infrared flux method and synthetic infrared colours to derive temperatures for G- and Ktype stars. Böhm-Vitense (1982) infers temperatures for spectral types A-F applying a temperature sensitive discontinuity of the spectral energy distribution at $1600 \AA$ A Jones et al. (1995) compared observed and synthetic spectra for transitions of water vapour in order to provide temperatures for M-dwarfs. 
M. Ammler et al.: What are the effective temperatures of T Tauri stars?, Online Material p 6

\section{Appendix B: Conversion tables}

Table B.1. The temperature conversion scales for dwarfs at spectral types G0-M 9. For details on the references see Table 2. Temperatures are given in $[\mathrm{K}]$.

\begin{tabular}{|c|c|c|c|c|c|c|c|}
\hline Spectral type & bessell7991 ${ }^{1}$ & ck79 ${ }^{2}$ & $\operatorname{djn} 87^{3}$ & hss94 & kh95 & luhman036 & tokunaga00 ${ }^{7}$ \\
\hline G0 & 6000 & 5900 & 5940 & 6026 & 6030 & & 5930 \\
\hline G1 & & 5830 & & & 5945 & & \\
\hline $\mathrm{G} 2$ & & 5770 & 5790 & 5860 & 5860 & & 5830 \\
\hline G3 & & & & & 5830 & & \\
\hline G4 & & & 5640 & & 5800 & & 5740 \\
\hline G5 & & 5660 & & 5754 & 5770 & & \\
\hline G6 & 5500 & & & & 5700 & & 5620 \\
\hline G7 & & & & & 5630 & & \\
\hline G8 & & 5450 & 5310 & 5572 & 5520 & & \\
\hline G9 & & & & & 5410 & & \\
\hline K0 & & 5240 & 5150 & 5248 & 5250 & & 5240 \\
\hline K1 & & 5110 & 4990 & 5082 & 5080 & & \\
\hline $\mathrm{K} 2$ & 5000 & 4950 & & 4898 & 4900 & & 5010 \\
\hline K3 & & 4780 & 4690 & 4732 & 4730 & & \\
\hline K4 & 4500 & 4580 & 4540 & 4592 & 4590 & & 4560 \\
\hline K5 & & 4400 & 4410 & 4395 & 4350 & & 4340 \\
\hline K6 & & 4200 & & & 4205 & & \\
\hline K7 & 4000 & 4000 & 4150 & 3999 & 4060 & & 4040 \\
\hline K7-M0 & & 3960 & & & & & \\
\hline K9 & & & 3940 & & & & \\
\hline M0 & 3800 & 3920 & 3840 & 3802 & 3850 & & 3800 \\
\hline M0.5 & & 3800 & & 3724 & & & \\
\hline M 1 & 3650 & 3680 & 3660 & 3648 & 3720 & 3680 & 3680 \\
\hline M 1.5 & & 3590 & 3590 & 3573 & & & \\
\hline M2 & 3500 & 3500 & 3520 & 3499 & 3580 & 3510 & 3530 \\
\hline M 2.5 & & 3430 & & & & & \\
\hline M3 & 3350 & 3360 & 3400 & 3350 & 3470 & 3350 & 3380 \\
\hline M 3.5 & & 3300 & & & & & \\
\hline M4 & 3150 & 3230 & 3290 & 3148 & 3370 & 3180 & 3180 \\
\hline M 4.5 & & & & & & & \\
\hline M 5 & 3000 & 3120 & 3170 & 2999 & 3240 & 2960 & 3030 \\
\hline M 5.5 & 2900 & 3040 & & & & & \\
\hline M 6 & 2800 & 2960 & 3030 & 2799 & 3050 & 2740 & 2850 \\
\hline M 6.5 & 2700 & & 2950 & & & & \\
\hline M7 & 2600 & & 2860 & & & 2620 & \\
\hline M 7.5 & 2450 & & & & & & \\
\hline M 8 & & & 2670 & & & 2500 & \\
\hline M9 & & & 2440 & & & 2300 & \\
\hline
\end{tabular}

${ }^{1}$ bessell7991 = combination of Bessell (1979) and Bessell (1991); ${ }^{2}$ ck79 = Cohen \& Kuhi (1979); ${ }^{3}$ djn87 = de Jager \& Nieuwenhuijzen (1987);

${ }^{4}$ hss94 = Hartigan et al. (1994); ${ }^{5}$ kh95 = Kenyon \& Hartmann $(1995) ;{ }^{6}$ luhman03 = Luhman et al. $(2003) ;{ }^{7}$ tokunaga00 = Tokunaga $(2000)$. 
M. Ammler et al.: What are the effective temperatures of T Tauri stars?, Online Material $p 7$

Table B.2. Same as Table B.1 for the non-dwarf luminosity classes. The reference abbreviations are appended with "g" for a giant scale, "sg" for a subgiant scale and "i" for an intermediate scale. See Table 2 for further details. Again temperatures are given in [K].

\begin{tabular}{|c|c|c|c|c|c|c|}
\hline Spectral type & bessell79g $\mathrm{g}^{1}$ & djn87sg ${ }^{2}$ & djn87g ${ }^{3}$ & perrin98g ${ }^{4}$ & luhman03i ${ }^{5}$ & luhman99g6 \\
\hline G0 & \multirow{10}{*}{5000} & 5640 & 5470 & & & \\
\hline G1 & & & & & & \\
\hline $\mathrm{G} 2$ & & 5460 & 5300 & & & \\
\hline G3 & & & & & & \\
\hline G4 & & 5280 & 5130 & & & \\
\hline G5 & & & & & & \\
\hline G6 & & & & & & \\
\hline G7 & & & & & & \\
\hline G8 & & 4940 & 4800 & 4930 & & \\
\hline G9 & & & & & & \\
\hline K0 & \multirow[t]{2}{*}{4750} & 4780 & 4660 & 4790 & & \\
\hline $\mathrm{K} 1$ & & 4620 & 4510 & 4610 & & \\
\hline $\mathrm{K} 2$ & 4500 & & & 4450 & & \\
\hline K3 & \multirow{2}{*}{4250} & 4340 & 4260 & 4270 & & \\
\hline K4 & & 4210 & 4150 & 4095 & & \\
\hline K5 & \multirow[t]{9}{*}{4000} & 4080 & 4050 & 3980 & & \\
\hline K6 & & & & & & \\
\hline K7 & & 3870 & 3870 & & & \\
\hline K7-M0 & & & & & & \\
\hline K9 & & 3700 & 3740 & & & \\
\hline M0 & & 3630 & 3690 & 3895 & & \\
\hline M0.5 & & & & & & \\
\hline M 1 & & 3510 & 3600 & 3810 & 3705 & 3800 \\
\hline M 1.5 & & 3460 & 3560 & & & \\
\hline M 2 & \multirow[t]{5}{*}{3750} & 3410 & 3540 & 3730 & 3560 & 3700 \\
\hline M 2.5 & & & & & & \\
\hline M 3 & & 3340 & 3480 & 3640 & 3415 & 3590 \\
\hline M 3.5 & & & & & & \\
\hline M 4 & & 3280 & 3440 & 3560 & 3270 & 3480 \\
\hline M 4.5 & \multirow[t]{3}{*}{3500} & & & & & \\
\hline M 5 & & 3220 & 3380 & 3420 & 3125 & 3370 \\
\hline M 5.5 & & & & & & \\
\hline M 6 & \multirow[t]{6}{*}{3250} & 3150 & 3330 & 3243 & 2990 & 3250 \\
\hline M 6.5 & & 3110 & 3300 & & & \\
\hline M 7 & & 3070 & 3270 & 3087 & 2880 & 3100 \\
\hline M 7.5 & & & & & & \\
\hline M 8 & & 2990 & 3240 & 2806 & 2710 & 2800 \\
\hline M9 & & 2920 & 3270 & & 2400 & 2650 \\
\hline
\end{tabular}

1 bessell79g = Bessell (1979); ${ }^{2}$ djn87sg=de Jager \& Nieuwenhuijzen (1987); ${ }^{3}$ djn87g=de Jager \& Nieuwenhuijzen (1987); ${ }^{4}$ perrin98g = Perrin et al. (1998); ${ }^{5}$ luhman03i $=$ Luhman et al. $(2003) ;{ }^{6}$ luhman99g $=$ Luhman $(1999)$. 